\title{
Discours
}

Revue de linguistique, psycholinguistique et

informatique. A journal of linguistics, psycholinguistics and computational linguistics

$21 \mid 2017$

Varia

\section{Portée propositionnelle et portée énonciative des adverbiaux cadratifs abstraits en fait et en réalité}

\section{Dennis Wandel}

\section{OpenEdition}

Journals

Édition électronique

URL : http://journals.openedition.org/discours/9384

DOI : 10.4000/discours.9384

ISSN : 1963-1723

Éditeur :

Laboratoire LATTICE, Presses universitaires de Caen

\section{Référence électronique}

Dennis Wandel, «Portée propositionnelle et portée énonciative des adverbiaux cadratifs abstraits en fait et en réalité ", Discours [En ligne], 21 | 2017, mis en ligne le 22 décembre 2017, consulté le 21 avril 2019. URL : http://journals.openedition.org/discours/9384 ; DOI : 10.4000/discours.9384 

Revue de linguistique, psycholinguistique et informatique

\section{Portée propositionnelle et portée énonciative des adverbiaux cadratifs abstraits en fait et en réalité}

Dennis Wandel

Linguistique française

Université de Neuchâtel

Dennis Wandel, «Portée propositionnelle et portée énonciative des adverbiaux cadratifs abstraits en fait et en réalité», Discours [En ligne], 21 | 2017, mis en ligne le 22 décembre 2017.

URL: http://journals.openedition.org/discours/9384

Titre du numéro: Varia

Coordination: Nicolas Hernandez \& Christian Raymond 



\title{
Portée propositionnelle et portée énonciative des adverbiaux cadratifs abstraits en fait et en réalité
}

\author{
Dennis Wandel \\ Linguistique française \\ Université de Neuchâtel
}

\begin{abstract}
Cet article propose une analyse des différents emplois des adverbiaux cadratifs en pratique, en réalité et en fait. Ces adverbiaux ont une fonction cadrative propositionnelle qui porte sur le contenu propositionnel de l'énoncé et qui l'introduit dans une certaine perspective. Mais dans certains emplois, en réalité et en fait peuvent avoir une portée cadrative énonciative. Leur portée cadrative ne concerne alors le contenu propositionnel qu'indirectement, car en fait elle indique l'intervention du locuteur et sert à qualifier le positionnement énonciatif de l'énoncé au niveau discursif. Cette hypothèse est vérifiée au moyen d'une étude sur corpus. Afin de distinguer les emplois à portée cadrative propositionnelle et ceux à portée cadrative énonciative, un codage opérationnel en fonction du mouvement discursif sousjacent est proposé: en partant d'un repérage manuel des «indicateurs contextuels » qui, en cooccurrence avec l'adverbial cadratif abstrait, signalent un changement de perspective au niveau du contenu, il est possible de coder les emplois à portée cadrative propositionnelle. La spécificité de ce type d'indicateurs contextuels en tant que cooccurrents des adverbiaux cadratifs abstraits est vérifiée. Dans le cas où aucun indicateur contextuel n'intervient dans le contexte discursif gauche de l'adverbial cadratif abstrait, il s'agit d'un emploi à portée cadrative énonciative.
\end{abstract}

Mots clés: adverbiaux cadratifs abstraits, en réalité, en fait, modèle triadique de représentation des sens modaux, portée cadrative propositionnelle, portée cadrative énonciative, cooccurrences, mouvement discursif

This paper presents an analysis of the different uses of the framing adverbials en pratique, en réalité and en fait. These adverbials have a framing function that relates to the propositional content of the utterance which is introduced from a specific perspective. In certain cases, however, en réalité and en fait can also have a framing function that concerns the enunciation. The scope of their framing function hence only indirectly concerns the propositional content and indicates, rather, an intervention by the speaker who qualifies the enunciative positioning of his utterance on the discourse level. This hypothesis is verified by a corpus study. In order to distinguish between uses framing the propositional content and uses framing the enunciation, an operational coding system is proposed based on the underlying discourse pattern: starting with a manual tracking of "contextual indicators" which, co-occurring with the abstract framing adverbial, indicate a change of perspective on the propositional level, it is possible to code uses framing the propositional content. The specificity of this type of contextual indicators as collocates of the framing adverbials is verified. However, when no such indicator is present in discourse in the left context of a framing adverbial, its framing function has an enunciative scope.

Keywords: abstract framing adverbials, en réalité, en fait, triadic model of representation of modal meaning, propositional scope, enunciative scope, co-occurrences, discourse pattern 


\section{Introduction}

Nous proposons une étude ${ }^{1}$ des adverbiaux cadratifs abstraits (AdvCA) en pratique, en réalité et en fait pour lesquels nous distinguons deux emplois. Selon le modèle triadique de représentation des sens modaux de Rossari (2016), ces emplois diffèrent en fonction de la cible de leur portée cadrative: l'énoncé ou l'énonciation. Notre travail teste un codage basé sur la présence d'indicateurs dans le contexte discursif gauche des AdvCA qui déclenchent, en cooccurrence avec l'AdvCA, un certain mouvement discursif dont paraît dépendre la portée cadrative de ces adverbes.

Détachés de la phrase d'accueil, donc dans une position extraprédicative ou exophrastique ${ }^{2}$, les AdvCA regroupent les informations dans le discours au sein de blocs d'information (définis comme cadres par Charolles [1997]). La cible de leur portée cadrative peut être le contenu propositionnel de l'énoncé ou l'énonciation (Charolles et Vigier, 2005). La portée cadrative d'un AdvCA est dérivée des indications sémantiques données par les composants de la forme. La préposition en a une affinité avec le pôle de la «thématicité» (Blumenthal, 2017) et manifeste «une préférence combinatoire pour certains termes généraux et abstraits» (Vigier, 2015: 245) tels que réalité, fait ou pratique pour créer des formes cadratives (voir aussi De Mulder et Amiot, 2013; Fagard et Combettes, 2013).

L'objectif principal de notre étude est une distinction des différents emplois cadratifs des AdvCA dans de différents mouvements discursifs. Nous distinguons deux emplois des AdvCA en fonction d'un seul critère - celui de la cible de leur portée cadrative. Dans la perspective de Roulet, reprise par exemple dans Rossari (1997), nous considérons comme «mouvement discursif» «une intervention qui se donne à un moment donné du discours comme autonome, se suffisant à elle-même» (Roulet, 1987: 113). Un discours consiste ainsi en des enchainements d'interventions interactionnelles et argumentatives. Nous définissons un «mouvement discursif» comme un ou plusieurs énoncés qui sont représentés d'une certaine manière dans la suite du discours en poursuivant un but rhétorique spécifique. Par exemple, dans une structure concessive ont lieu deux mouvements discursifs qui déclenchent un contraste. Il en résulte deux représentations différentes d'énoncés: un premier énoncé est mis à l'arrière-plan discursif (par une forme telle que certes) tandis qu'un second énoncé, qui suit le premier, est mis à l'avant-plan discursif-marqué par mais et/ou un connecteur reformulatif tel que en réalité (Rossari, 2015).

1. Cette étude s'inscrit dans le cadre du projet de recherche financé par le Fonds national suisse (FNS $\mathrm{n}^{\circ}$ 100012_159458) intitulé «La représentation du sens modal et de ses tendances évolutives dans deux langues romanes: le français et l'italien» (université de Neuchâtel), dirigé par Corinne Rossari et en collaboration avec Annalena Hütsch, Claudia Ricci et Dennis Wandel.

Nous tenons à remercier les relecteurs anonymes ainsi que Claudia Ricci et Corinne Rossari pour leurs nombreux commentaires constructifs qui ont permis de prendre en compte des aspects qui n'avaient pas reçu d'attention dans les versions antérieures de cet article.

2. Au sens de Guimier $(2007,1996)$. Par la suite nous utilisons le terme «extraprédicatif» pour désigner tous les emplois exophrastiques et endophrastiques qui ne sont pas intraprédicatifs. 
Selon le type de mouvement discursif dans lequel les AdvCA interviennent, leur portée cadrative concerne soit le contenu propositionnel d'un énoncé, soit l'énonciation ${ }^{3}$, donc l'apparition d'un énoncé.

Les deux types d'emplois à portées cadratives différentes sont illustrés par les exemples suivants tirés du «Corpus des variétés nationales du français» (CoVaNa-FR; Diwersy, 2014) nommé «Varitext» sur la plateforme BTLC.Primestat (Diwersy, 2013):

[1] En apparence, c'est donc la continuité par rapport à la première saison. En réalité, les scénaristes et les réalisateurs ont changé.

[2] Astérix aux Jeux olympiques est un ouvrage en trois actes si totalement hétérogènes qu'ils semblent avoir été mis en images par trois équipes différentes. En fait, deux, puisqu'il transpire de partout la présence d'un duo de réalisateurs [...].

[3] La peine de mort a été légalement abolie au Canada en 1976. En pratique, elle n'était plus appliquée depuis 1962.

[4] Aujourd'hui encore, un proche du président évoque le risque d'un «soulèvement» en cas de signature [de la loi]. En réalité, des négociations ont lieu.

[5] Tout dépend du règlement. En fait, l'anomalie législative est un effet secondaire du rejet de la onzième révision de l'AVS en mai 2004.

Dans les exemples [1], [2] et [3], les AdvCA portent sur le contenu propositionnel des énoncés qu'ils introduisent. En réalité, en fait et en pratique ont ce que nous appelons une "portée cadrative propositionnelle» (PCP) qui représente l'énoncé suivant comme relevant du cadre de la «réalité», des «faits» ou de la «pratique». Notons que dans ces exemples la PCP des AdvCA coïncide avec la présence d'un autre marqueur qui fait référence à un autre cadre qui est mis en contraste avec le cadre introduit par l'AdvCA. Ces marqueurs sont l'adverbial cadratif en apparence (exemple [1]), le verbe d'état sembler (exemple [2]) représentant aussi un cadre qui appartient aux «apparences», et l'adverbe cadratif légalement (exemple [3]). Dans les trois exemples, on constate un changement de perspective au niveau du contenu marqué par l'enchaînement de plusieurs cadres thématiques différents.

Dans les exemples [4] et [5], les AdvCA portent sur l'énonciation. Ils ont ce que nous appelons une "portée cadrative énonciative» (PCE). Ils ne concernent le contenu propositionnel des énoncés qu'indirectement. L'accent est mis sur leur fonction discursive qui permet au locuteur de définir la perspective énonciative (Rossari, 1997: 9) dans laquelle les énoncés concernés sont représentés. En réalité

3. Nous utilisons la définition de Ducrot (1980) pour qui l'énonciation n'est pas l'acte même d'énoncer, mais le fait que l'apparition d'un énoncé a lieu, dont le locuteur est conscient et sur lequel il peut faire un commentaire. "J'appellerai “énonciation” le fait même que l'énoncé ait été produit, l'événement historique constitué par son apparition [...]. Il s'agit d'une pure occurrence d'une phrase de la langue réalisée, sous une forme particulière, en un point et un moment particuliers» (Ducrot, 1980: 30). 
et en fait attirent l'attention de l'interlocuteur sur la façon dont l'énoncé introduit par l'AdvCA est représenté par rapport à l'énoncé précédent. L'énoncé introduit par l'AdvCA est alors mis à l'avant-plan du discours au détriment de l'énoncé précédent qui est rétrospectivement mis à l'arrière-plan, ainsi représenté comme étant moins pertinent comme argument (voir la fonction reformulative telle qu'elle est définie dans Rossari [1997]). Ici, il ne s'agit pas d'une opposition de cadres thématiques au niveau du contenu propositionnel comme dans [1], [2] et [3], mais d'un changement de perspective énonciative qui est issu d'une représentation différente dans le discours de l'énoncé introduit par l'AdvCA par rapport à l'énoncé précédent (Rossari, 1997: 9). On peut parler d'un emploi en tant que «connecteur reformulatif» (voir Rossari, 1997). Pour en pratique, un tel emploi à portée énonciative n'est pas attesté.

Dans la première partie de cet article, nous donnons un aperçu des travaux existants qui thématisent les différentes fonctions cadratives des AdvCA. Puis, nous proposons une représentation des emplois à $\mathrm{PCP}$ et à $\mathrm{PCE}$ des AdvCA par le modèle triadique de Rossari (2016). Les différents emplois des AdvCA sont considérés comme un cas de polyvalence : la portée cadrative représente le «composant sémantique stable» d'un AdvCA dans tous ses emplois, mais sa cible varie selon le contexte discursif dans lequel est utilisé l'adverbial. Pour les exemples [1], [2] et [3], où les AdvCA ont une PCP, à savoir une portée sur le contenu des énoncés, nous avons constaté que dans leur contexte discursif gauche se trouvent d'autres formes cadratives qui introduisent un cadre différent ou opposé (telles que en apparence, sembler et légalement). Afin de proposer un codage opérationnel des différents emplois des AdvCA en fonction du mouvement discursif dans lequel ils interviennent, il nous faut analyser le contexte discursif. Nous déterminons si la présence d'«indicateurs contextuels» signalant une perspective différente de celle introduite par l'AdvCA au niveau du contenu converge toujours avec une PCP de l'AdvCA. Puis, nous appliquons ce codage à 50 occurrences de en réalité et de en fait pour déterminer s'il existe des cas où aucun indicateur contextuel n'est présent dans le contexte gauche de l'AdvCA, cela étant un indice d'un emploi à PCE qui met en contraste deux perspectives énonciatives différentes (exemples [4] et [5]). Outre un tri manuel des emplois effectué sur des échantillons de 50 occurrences de chaque adverbial, nous utilisons un repérage automatique sur corpus des cooccurrents spécifiques des AdvCA afin de savoir si ce type d'indicateurs apparait typiquement dans le contexte discursif gauche des trois AdvCA. Les résultats de cette analyse sur corpus nous permettent d'évaluer la fiabilité du codage proposé. Enfin, nous testons le même codage pour les adverbiaux cadratifs référentiels dans la réalité, dans les faits et dans la pratique qui, comme en pratique, ont toujours une PCP et dont le contexte gauche devrait donc toujours contenir des indicateurs contextuels d'une perspective différente au niveau du contenu, si notre codage est fiable.

\section{2. Études existantes sur en fait, en réalité et en pratique}

Les appellations utilisées dans les travaux existants pour distinguer les différentes portées des formes cadratives varient et manquent parfois d'une définition claire 
qui permettrait une distinction nette entre les différents emplois. Mais ces travaux ont une chose en commun: la plupart d'entre eux sont fondés sur la «théorie des cadres» proposée par Charolles (1997). Dans les études qui ne mentionnent pas explicitement les AdvCA en pratique, en réalité et en fait, mais qui proposent une classification et description générale des cadres, ce type de formes adverbiales fait partie du groupe des introducteurs de cadres de discours (Charolles, 1997; Ferret et al., 2001; Vigier, 2003). Un certain nombre d'études des formes cadratives se basent sur une analyse sur corpus: Charolles et Pietrandrea (2012) étudient les fonctions modales et discursives de en réalité, Vigier (2015) se concentre sur les prépositions en, dans et dedans et propose une analyse sémantique, statistique et combinatoire en diachronie de leurs fonctions dans des syntagmes prépositionnels, Prévost (2003) analyse les liens entre topiques du discours et formes cadratives, Charolles et Vigier (2005) s'intéressent à la portée cadrative des adverbiaux en position préverbale et à leur contribution à l'organisation des discours, Porhiel (2005) analyse la formation des séquences thématiques dans le discours par des introducteurs thématiques, et Ferret et al. (2001) testent les limites d'un repérage automatisé des structures thématiques d'un texte.

Dans les travaux qui ont comme objet d'étude la polyvalence des adverbiaux en réalité et en fait, les appellations choisies pour leurs différents emplois varient également. Mais ces descriptions fonctionnelles ont en commun le constat que, dans des emplois extraprédicatifs, les AdvCA ne concernent souvent pas seulement le contenu propositionnel de l'énoncé qu'ils introduisent, mais ont aussi des fonctions discursives reliant cet énoncé à un ou à plusieurs énoncés précédents et/ou suivants.

Charolles (1997: 41) pointe sur ce fait en indiquant que «ces marqueurs n'expriment pas ou, pour le moins, pas directement et pas seulement, des relations évaluables en termes de vérité/fausseté parce qu'ils ne font pas que coder des rapports entre des états de choses existant indépendamment des locuteurs». Pour l'auteur, il s'agit de cadres qui désignent des «univers d'énonciation» qui incluent «dans leur portée des questions, des énoncés modalisés ainsi que des connecteurs» (Charolles, 1997: 47). Pourtant, cette fonction de en réalité reste directement liée aux propositions adjacentes: «En réalité, de par son sémantisme, indique clairement un changement de cadre d'appréciation, seulement ce changement joue à un autre niveau. L'opposition se fait avec un univers parent, à savoir l'univers des apparences, sous lequel le lecteur est invité à intégrer rétrospectivement la totalité du texte [...]» (Charolles, 1997: 42).

Déjà, la définition d'une classe de formes ayant une fonction «reformulative» par Roulet (1987) s'adosse sur la description de leurs fonctions discursives. Pour l'auteur, il s'agit d'une «reformulation» «d'un premier mouvement discursif (ou d'un implicite)» qui est non paraphrastique, «car l'énonciateur tente de mieux satisfaire à la complétude interactive en présentant l'intervention principale comme une nouvelle formulation, liée à un changement de perspective énonciative indiqué par 
le connecteur» (Roulet, 1987: 115), mais sans «reformuler» une proposition par une véritable paraphrase (voir ce cas de reformulation paraphrastique dans Gülich et Kotschi [1983]).

Iordanskaja et Mel'čuk (1995) ne prennent en compte que les emplois en tant que connecteurs des AdvCA en fait et en réalité dans une position détachée de la phrase d'accueil. Pour les auteurs, il s'agit de lexies non descriptives avec une fonction «signalative, locutive et discursive» qui indique que l'énoncé introduit par en fait ou en réalité est une réfutation ou précision de l'information donnée par un énoncé précédent.

Rossari (1997) propose une analyse des fonctions des adverbiaux en réalité et en fait en tant que «connecteurs reformulatifs» présentant une fonction discursive qui déclenche un «changement de perspective énonciative émanant d'une réinterprétation du mouvement discursif antécédent: le locuteur, suite à une première formulation donnée comme autonome et donc formant un premier mouvement discursif, en ajoute une seconde qui vient englober la première en la subordonnant rétroactivement» (Rossari, 1997: 9). La fonction des connecteurs reformulatifs en fait et en réalité est d'introduire une nouvelle perspective énonciative.

De même, mais selon une analyse davantage centrée sur le contenu, Blumenthal (1996) atteste une polyvalence de la locution en fait, caractérisée comme un connecteur qui peut être un «rhématisateur phatique», un marqueur d' «opposition entre le faux et le vrai» et un connecteur ayant une fonction argumentative où «la phrase contenant en fait appuie un énoncé précédent dont le contenu s'oppose à un autre énoncé» (Blumenthal, 1996: 267).

Une idée similaire est décrite dans Charolles et Vigier (2005: 24). Les auteurs analysent la portée cadrative des adverbiaux en position préverbale afin de rendre compte de la fonction organisationnelle qu'ils peuvent avoir au-delà de la phrase d'accueil (Charolles et Vigier, $2005:$ 9). Cette fonction cadrative est mise en contraste avec la fonction reformulative de en réalité quand ce connecteur mentionne «une connaissance à laquelle les autres n'ont pas accès».

Charolles et Pietrandrea (2012) proposent une étude sur corpus de l'usage de l'adverbial en réalité où ils opposent essentiellement deux fonctions différentes. D'un côté, en réalité fonctionne comme un adverbial détaché de la phrase qui «signale que le contenu de celle-ci s'oppose à celui d'une ou de plusieurs phrases mentionnée(s) précédemment. L'opposition porte sur la valeur de vérité des situations dénotées par les phrases: en réalité indique que la situation à laquelle fait allusion la phrase en tête de laquelle il figure est avérée, au contraire de celles mentionnées dans la complétive qui précède qui ne sont vraies qu'en apparence» (Charolles et Pietrandrea, 2012: 111). De l'autre côté, l'adverbial a une multitude de fonctions discursives du fait que «même si en réalité se rapproche des connecteurs oppositifs (réfutatifs ou concessifs) et reformulatifs, il peut aussi se rapprocher des connecteurs signalant d'autres relations de discours» (Charolles et Pietrandrea, 2012: 112). 


\section{Portée cadrative propositionnelle et portée cadrative énonciative des adverbiaux cadratifs abstraits}

Comme indiqué dans l'introduction, nous faisons l'hypothèse que les $\operatorname{AdvCA}$ en réalité et en fait ont une portée cadrative qui peut avoir plusieurs cibles: a) le contenu des énoncés; b) l'énonciation dans le cas où ils concernent la représentation des énoncés dans la suite du discours. Par contraste, en pratique, qui est un AdvCA formé par les mêmes composants que en réalité et en fait (préposition en + nom abstrait désignant un cadre abstrait), ne partage que la portée cadrative qui concerne le contenu. Nous utilisons le terme de «portée» défini dans Charolles et Vigier (2005: 15) (voir aussi: Combettes, 2005): «En linguistique, la portée d'une UP [unité à portée] est en général définie comme le (ou les) constituant(s) de l'énoncé sur lequel (lesquels) cette unité exerce une influence sémantique». Les auteurs entendent par "portée cadrative» «l'ensemble des phrases qui appartiennent toutes à un même cadre de discours» (Charolles et Vigier, 2005: 18). Nous distinguons deux types de portée cadrative en fonction de la cible de cette portée: une portée cadrative propositionnelle (PCP) et une portée cadrative énonciative (PCE).

Pour notre analyse des emplois cadratifs des AdvCA, nous utilisons un modèle théorique qui stipule que des formes modales peuvent porter soit sur une ou plusieurs propositions, soit sur l'énonciation (voir Ducrot, 1980). Le modèle triadique de Rossari (2016) décrit les différents emplois d'une forme modale en fonction de trois paramètres que nous pouvons définir ainsi: le «composant sémantique stable», sa «portée» soit «propositionnelle», soit «énonciative», et la «motivation rhétorique». La motivation rhétorique influence l'actualisation de la «portée»du «composant sémantique stable» de la forme quand elle porte sur l'énonciation et non sur le contenu de l'énoncé. Le modèle triadique représente les différentes valeurs sémantiques d'une forme modale par différentes actualisations d'un unique «composant sémantique stable». C’est pourquoi, selon ce modèle, les différents emplois des AdvCA ne font pas preuve d'une polysémie, mais d'une polyvalence.

En appliquant ce modèle, nous précisons le terme de «portée cadrative» défini par Charolles et Vigier (2005) en distinguant deux types de «portée cadrative». D’un côté, les AdvCA en pratique, en réalité et en fait partagent une "portée cadrative propositionnelle» (PCP) qui est directement déduite de leur «composant sémantique stable» (voir les exemples [1], [2] et [3] dans l'introduction). Ce «composant sémantique» est «stable» car il est à l'origine de tout emploi cadratif d'un AdvCA. La portée cadrative est ainsi inscrite dans la forme: elle est issue de la combinaison des indications sémantiques données par la préposition en et par le nom qui désigne un concept abstrait (réalité, fait, pratique). De l'autre côté, contrairement à en pratique, en réalité et en fait peuvent aussi avoir une «portée cadrative énonciative» (PCE) quand les indications sémantiques tirées du même «composant sémantique stable» ne portent plus qu'indirectement sur le contenu propositionnel de l'énoncé. Dans ce cas, elles portent sur l'énonciation (voir les exemples [4] et [5] dans l'introduction). La portée cadrative inscrite dans le «composant sémantique stable» est actualisée au 
niveau discursif afin de contraster la manière dont le locuteur représente deux énoncés en mettant un premier énoncé à l'arrière-plan discursif et un deuxième énoncé, qui suit le premier, à l'avant-plan discursif. Les différents emplois des AdvCA analysés dans les études existantes présentées dans la deuxième partie résultent ainsi d'une polyvalence et non d'une polysémie des AdvCA. C'est la cible de la portée cadrative qui change en fonction du contexte discursif, et plus précisément du mouvement discursif dans lequel intervient l'AdvCA.

Illustrons cette idée avec quelques exemples. Les trois AdvCA ont une PCP qui active leur composant sémantique stable avec l'effet de désigner un cadre thématique pour l'énoncé qu'ils introduisent:

[6] Je crois qu'on a tendance à lancer de grands débats en oubliant de faire avancer des réformes dont la nécessité en pratique, est patente et qui prennent beaucoup de retard.

[7] À quelques mètres, au bord des rails, un homme ivre. On dirait qu'il rigole. En fait, il ressasse ses angoisses. En un instant, le malheureux se jette sur les voies.

[8] En apparence, c'est donc la continuité par rapport à la première saison. En réalité, les scénaristes et les réalisateurs ont changé.

Il est possible de paraphraser, avec des résultats plus ou moins naturels, tous ces emplois à $\mathrm{PCP}$ des AdvCA par les syntagmes prépositionnels référentiels dans la pratique, dans la réalité et dans les faits dont la portée cadrative est sémantiquement proche de la PCP des AdvCA et toujours référentielle (voir partie 8 de l'article):

[9] Je crois qu'on a tendance à lancer de grands débats en oubliant de faire avancer des réformes dont la nécessite, dans la pratique, est patente et qui prennent beaucoup de retard.

[10] À quelques mètres, au bord des rails, un homme ivre. On dirait qu'il rigole. Dans les faits, il ressasse ses angoisses. En un instant, le malheureux se jette sur les voies.

[11] En apparence, c'est donc la continuité par rapport à la première saison. Dans la réalité, les scénaristes et les réalisateurs ont changé.

Toutefois, ce test de paraphrase ne semble pas être opérationnel à des fins de distinction entre la PCP et la PCE des AdvCA, car l'acceptabilité de la paraphrase et l'interprétation dépendent trop de l'intuition du codeur et de l'exemple concret. En revanche, les trois exemples [6], [7] et [8] ouvrent une autre piste pour un possible codage des emplois des AdvCA avec une PCP: le fait de trouver des formes indiquant une perspective différente, au niveau du contenu, de celle introduite par l'AdvCA. Dans l'exemple [6], le cadre introduit par en pratique est opposé à un cadre de «croyance» marqué par le verbe d'état croire. De même, en fait (exemple [7]) introduit un cadre de «faits» qui est opposé à un autre cadre pouvant être déduit 
de la phrase on dirait que: celle-ci exprime en effet une «incertitude» par rapport au contenu présenté, ainsi qu'un cadre que l'on pourrait décrire comme celui des «apparences». Pour en réalité (exemple [8]), ceci est encore plus évident: l'énoncé introduit par l'AdvCA en réalité est opposé au cadre des «apparences» introduit dans l'énoncé précédent par l'adverbial en apparence. Ces observations concordent avec l'étude menée par Charolles et Pietrandrea (2012) qui remarquent une corrélation entre la cooccurrence mutuelle de deux formes cadratives indiquant deux cadres opposés, par exemple en apparence et en réalité, et une portée cadrative (évidentielle) portant sur le contenu dans ce type d'emplois des AdvCA:

En réalité connecteur gardant un potentiel cadratif est également très souvent utilisé (au moins à l'écrit) pour structurer l'information discursive en relation avec en apparence, ce qui tend au contraire à favoriser le maintien de son sens évidentiel.

(Charolles et Pietrandrea, 2012: 139)

En revanche, dans certains emplois de l'AdvCA en réalité, la PCP de son composant sémantique stable n'est pas accessible. Une PCP étant exclue logiquement par le contexte, il s'agit d'un emploi où la forme a une PCE et marque un changement de perspective énonciative en tant que connecteur reformulatif:

[12] M. Saakachvili a certes répété qu'il était «hors de question» d'ouvrir un débat sur le sujet. En réalité, il est ouvert depuis la chute de l'Union soviétique...

Cet exemple ne permet aucune lecture qui suggérerait que en réalité introduit un cadre de la «réalité» au niveau du contenu. Le composant sémantique stable de l'adverbial ne porte plus directement sur le contenu propositionnel et ainsi ne désigne plus le cadre de validité pour le contenu de l'énoncé qu'il introduit. L'AdvCA a une PCE qui actualise le composant sémantique stable pour qualifier l'énonciation: l'énoncé introduit par en réalité est présenté par le locuteur comme étant mis à l'avant-plan de son discours tandis que l'énoncé précédent (marqué par certes) est mis à l'arrière-plan discursif. L'AdvCA marque une différence de pertinence entre arguments.

De même, dans certains emplois de en fait, une PCP de l'AdvCA est exclue. Le composant sémantique stable de l'adverbial est alors actualisé au niveau énonciatif pour attirer l'attention de l'interlocuteur sur l'information que le locuteur veut mettre en avant dans son discours:

[13] «La nouvelle console utilisera une unité centrale de 32 bits capable de traiter quatre fois plus de données que la Game Boy Color, 17 fois plus rapidement, et affichera 10 fois plus de couleurs simultanément. Son écran à cristaux liquides est une fois et demie plus large que celui de sa petite sœur et offrira une définition supérieure de $66 \%$. En fait, la Game Boy Advance est capable de fournir une image plus nette que les consoles actuelles de 32 bits et de 634 bits jouant sur un téléviseur de 27 pouces», précise Peter MacDougall. 
Dans les exemples [12] et [13], les AdvCA en fait et en réalité fonctionnent en tant que connecteurs reformulatifs. Cette fonction a été examinée par Rossari (1997, 1992) comme une fonction discursive des adverbiaux de fait, en fait et en réalité. L'adverbial reformulatif marque une précision ou une altération de l'information déjà présentée dans un énoncé précédent sans nécessairement la réfuter, mais en en déclenchant une réinterprétation. Une réinterprétation ne signifie pas une opposition «faux/vrai» qui est caractéristique de l'emploi à PCP (voir Blumenthal, 1996). Rossari (1992) caractérise la fonction de en fait dans cet emploi ainsi :

Le point de vue introduit par en fait est présenté comme établissant un fait nouveau qui s'écarte plus ou moins fortement d'un état de choses exprimé dans un point de vue antécédent, d'où la prise de distance de la part du locuteur vis-à-vis de ce point de vue. La légitimité de ce nouveau point de vue est assurée par son adéquation aux faits enregistrés dans la mémoire discursive du locuteur.

(Rossari, 1992: 153)

Nous en concluons que les AdvCA marquent un changement de perspective dans tous leurs emplois. Dans les emplois à PCP, l'AdvCA introduit un cadre abstrait (concernant la validité des états de choses présentés) qui contribue à l'enchaînement argumentatif du discours en fonction de blocs d'information. Ce fait sera exploité par notre étude sur corpus dans l'objectif d'établir un codage permettant l'identification de ces emplois à PCP des AdvCA en fonction d'indicateurs contextuels qui signalent une opposition de perspectives au niveau du contenu. Dans les emplois à PCE, l'AdvCA marque également un changement de perspective, mais au niveau énonciatif, où il s'agit d'une représentation différente des énoncés par le locuteur. Alors que l'AdvCA garde sa portée cadrative, il n'introduit pas de cadre thématique pour un énoncé. L'AdvCA à PCE marque l'intervention du locuteur qui vise à représenter ses énoncés d'une certaine manière: l'AdvCA met l'énoncé qu'il introduit à l'avant-plan du discours, indiquant ainsi la plus grande pertinence de son énonciation par rapport aux énonciations précédentes, qui sont rétrospectivement mises à l'arrière-plan discursif (voir Rossari, 1997). Ceci ne signifie pas que ce changement de perspective énonciative n'est pas parfois accompagné d'un changement de perspective au niveau du contenu, changement qui peut être marqué par d'autres formes cadratives à PCP et/ou qui peut être déduit du contexte.

\section{Corpus, plateforme et fréquence d'occurrence des adverbiaux cadratifs abstraits}

Pour tester notre hypothèse, selon laquelle l'analyse du contexte discursif permet de distinguer les emplois à PCP et les emplois à PCE des AdvCA dans la plupart des cas, nous avons recours à une étude sur corpus. Nous utilisons le «Corpus des variétés nationales du français» (CoVaNa-FR) (Diwersy, 2014) qui contient des textes de presse nationale de onze pays francophones (Europe, Afrique et Canada). La presse 
nationale représente un genre discursif à vocation d'information qui contient un grand nombre de textes argumentatifs censés introduire des perspectives différentes. Le corpus est interrogeable sur la plateforme BTLC.Primestat (Diwersy, 2013) dont nous utilisons les fonctionnalités suivantes: la recherche des mots dans le contexte (KWIC = keyword in context) pour l'analyse du contexte des AdvCA, le repérage des cooccurrents spécifiques des AdvCA et l'affichage des fréquences d'occurrences dans le concordancier.

Notre corpus de travail est constitué de trois sections du corpus CoVaNa-FR: les sections de presse française, suisse et canadienne. Il contient une ou deux années de deux journaux nationaux de chaque pays: Le Devoir et Le Soleil pour le Québec (53,5 millions de mots), Le Figaro et Le Monde pour la France (53,3 millions de mots), Le Temps et La Tribune de Genève pour la Suisse (28 millions de mots). Notre corpus compte ainsi environ 135 millions de mots, ce qui permet de repérer un nombre suffisant d'occurrences des trois AdvCA qui sont en effet relativement peu fréquents (tableau 1).

\begin{tabular}{|l|r|r|r|r|r|r|}
\hline $\begin{array}{l}\text { Corpus: } \\
134935489 \text { mots }\end{array}$ & en pratique & en réalité & en fait & $\begin{array}{l}\text { dans la } \\
\text { pratique }\end{array}$ & $\begin{array}{l}\text { dans la } \\
\text { réalité }\end{array}$ & $\begin{array}{l}\text { dans les } \\
\text { faits }\end{array}$ \\
\hline Fréquence absolue & 1209 & 2409 & 7531 & 382 & 449 & 1120 \\
\hline $\begin{array}{l}\text { Fréquence relative } \\
\text { (par 1 million) }\end{array}$ & 8,96 & 17,85 & 55,81 & 2,83 & 3,33 & 8,30 \\
\hline
\end{tabular}

Tableau 1 - Fréquences relatives et absolues des AdvCA (et des formes cadratives référentielles pour comparaison, voir partie 8) dans le corpus de travail (134935489 mots; presse suisse, canadienne et française dans le corpus $\mathrm{CoVaNa}-\mathrm{FR})^{4}$

On observe une différence de fréquence entre en pratique d'un côté et en réalité et en fait de l'autre côté qui sont environ deux et six fois plus fréquents. Par contraste, l'AdvCA référentiel dans la pratique est aussi fréquent que dans la réalité, et dans les faits est seulement environ trois fois plus fréquent que les deux autres. Nous supposons que cette différence est en partie issue du fait que en pratique n'a que des emplois à $\mathrm{PCP}$ (tout comme les AdvCA référentiels formés sur dans), tandis que en réalité et en fait ont aussi des emplois à $\mathrm{PCE}$, ce qui potentiellement augmente le nombre d'emplois possibles. Cette hypothèse est vérifiée dans la partie suivante au moyen d'une étude sur corpus. Nous testons un codage des emplois à PCP en fonction de la présence d'indicateurs contextuels d'une perspective différente de celle introduite par l'AdvCA, afin de savoir si ce codage permet de distinguer les emplois à $\mathrm{PCP}$ et à $\mathrm{PCE}$ de en réalité et en fait.

4. Une requête restreinte a permis d'exclure des occurrences non pertinentes (voir explications ci-après). 


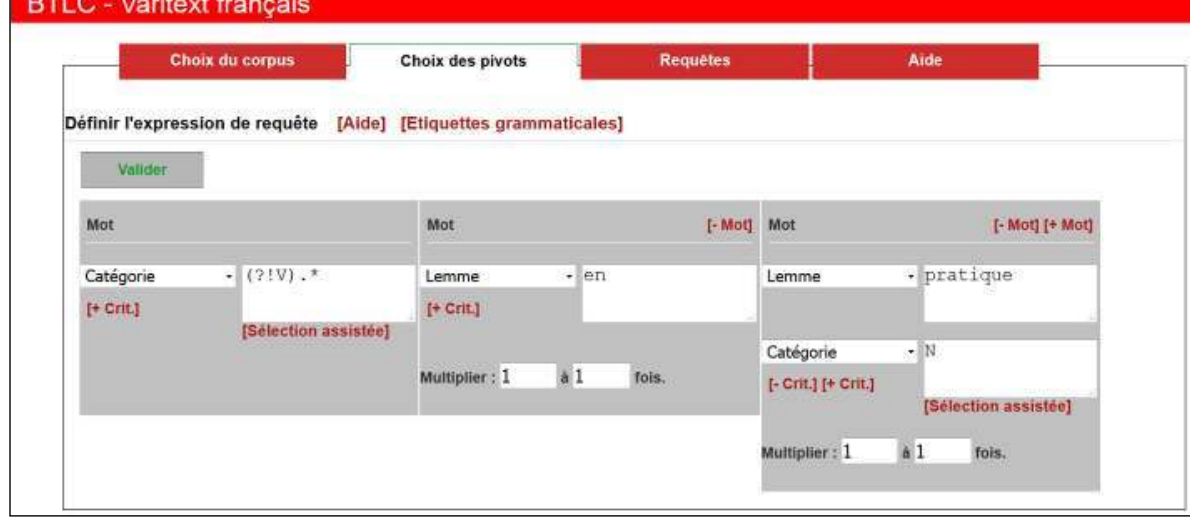

Figure 1 - Paramétrage des requêtes pour les emplois extraprédicatifs des AdvCA qui exclut les verbes occupant la position directement avant l'AdvCA: exemple de en pratique

Le paramétrage de toutes les analyses sur corpus que nous effectuons pose quelques problèmes: pour en fait, il est nécessaire de définir fait comme «nom» (catégorie) afin d'exclure les formes de la troisième personne du singulier ainsi que du participe passé du verbe faire. En outre, les trois AdvCA ont des emplois intraprédicatifs du type verbe $+A d v C A$ qui ne nous intéressent pas ici (pour donner un exemple de l'impact de ce bruit: une simple requête de la séquence $e n+$ fait relèverait six emplois intraprédicatifs sur 50 occurrences). Nous nous limitions à l'emploi extraprédicatif. La requête la plus apte à exclure la plupart des emplois intraprédicatifs sans être trop complexe est celle présentée dans la figure 1.

L'exclusion de tous les verbes intervenant directement avant l'AdvCA limite les exemples intraprédicatifs, même si certains, d'un nombre restreint, sont malgré tout pris en compte par le concordancier (3778 occurrences de en réalité sans exclusion des verbes avant l'AdvCA contre 2468 avec l'exclusion des verbes précédant l'AdvCA). On trouve néanmoins un certain nombre d'occurrences du type transformer leurs promesses en réalité, où d'autres éléments interviennent entre le verbe et en réalité. La situation est similaire pour en fait. Une idée de la répartition des emplois extra- et intraprédicatifs dans les résultats de cette requête sera donnée dans le cadre de nos analyses dans les sections suivantes. La requête formulée exclut ainsi la plupart des emplois intraprédicatifs de en fait et de en réalité ainsi que les formes conjuguées du verbe faire telles que il en fait trop, mais aussi certains emplois qui seraient pertinents du type il arrive en fait à... (emploi extraprédicatif). En revanche, un exemple pertinent tel que $i l$ n'arrive pas en fait à... se trouve dans les résultats car le verbe n'intervient pas directement avant la forme en fait. Il serait possible de préciser la requête encore en y ajoutant des paramètres plus complexes, mais nos tests ont montré qu'à partir 
de ce moment-là elle exclut plus d'emplois pertinents extraprédicatifs qu'elle ne peut exclure d'emplois intraprédicatifs.

\section{Repérage des «indicateurs contextuels» anticipant un changement de perspective au niveau du contenu}

Une distinction entre la PCP et la PCE des AdvCA ne peut pas être faite automatiquement en utilisant l'annotation du corpus CoVaNa-FR, car il n'existe pas d'étiquettes sémantiques pour les différents emplois. Il nous faut établir un codage opérationnel. Ferret et al. (2001) repèrent des structures thématiques (définies dans la perspective de Charolles [1997]) dans des textes en fonction de critères statistiques et linguistiques. Ils proposent «une reconnaissance de parties de texte liées à un même sujet [qui] est alors fondée sur la distribution des mots et leur récurrence» (Ferret et al., 2001: 166). Le cas où un mot est répété dans une zone limitée du texte est significatif pour l'identification du thème d'une partie du texte. Les auteurs combinent cette méthode statistique avec une méthode linguistique basée sur une généralisation de «la notion de répétition de termes», car «la cohésion thématique d'un texte se traduit par l'utilisation de termes faisant référence à une même entité par l'emploi de synonymes, d'hyponymes, de mots liés sémantiquement ou appartenant au même domaine» (Ferret et al., 2001: 167).

Afin de proposer un codage de la PCP des AdvCA en fonction d'un critère opérationnel, nous prenons en compte les déductions faites dans la partie précédente en analysant systématiquement le contexte discursif gauche des AdvCA pour trouver des indicateurs contextuels signalant une perspective différente, au niveau du contenu, de celle de l'AdvCA (voir aussi Charolles et Pietrandrea, 2012: 122-123). Dans les cas où au moins un tel indicateur intervient dans le contexte gauche de l'AdvCA (et lorsqu'une relation directe entre AdvCA et indicateur est perceptible au niveau discursif), les formes déclenchent ensemble un changement de perspective au niveau du contenu et l'AdvCA a une PCP. Ceci reprend l'idée que les formes cadratives enchainent des blocs d'information dans la suite du discours en indiquant à chaque reprise un changement de perspective (Charolles, 1997), par la précision d'un cadre ou l'introduction d'un nouveau cadre. En même temps, ce changement de perspective au niveau du contenu indique un certain mouvement discursif qui paraît être typique de l'emploi à PCP des AdvCA. Selon Charolles (1995: 128), il existe un ensemble hétérogène «d'outils relationnels de nature sémantico-pragmatique qui, en quelque sorte, complètent le système des relations distributionnelles et positionnelles de caractère syntaxique ainsi que le dispositif logico-énonciatif (thème/propos)». L'auteur distingue quatre classes de marqueurs (liste tirée de Charolles, 1995: 128):

1. les connecteurs qui indiquent des relations fonctionnelles entre les contenus propositionnels et/ou les actes illocutionnaires qui leur sont associés (relations du type: justification, opposition, consécution, etc.); 
2. les différentes formes d'anaphores qui assurent des solidarités référentielles (coréférence, associativité, etc.) entre certains constituants des énoncés et qui donnent naissance à des chaînes de référence;

3. les expressions introductrices de cadres de discours qui délimitent des domaines ou cadres (temporels, spatiaux, modaux, etc.) s'étendant parfois sur de vastes séquences;

4. les marques configurationnelles (alinéas, organisateurs métadiscursifs) qui délimitent au sein du continuum textuel des ensembles présentés par le locuteur comme constituant une ou plusieurs unités en regard d'un certain critère dispositionnel.

En principe, tous ces types de marqueurs sauf les «marques configurationnelles» opèrent au niveau des contenus des énoncés (à l'exception de certaines formes cadratives qui portent sur l'énonciation d'après Charolles) et, grâce à leur fonction argumentative, discursive et/ou cadrative, peuvent alors introduire une perspective différente de celle marquée par l'AdvCA à PCP. Nous n'excluons aucune de ces classes de marqueurs de notre codage.

Nous cherchons surtout des formes qui font référence à un cadre ou à une perspective qui sont différents de ceux introduits par les AdvCA, donc surtout des «expressions introductrices de cadres de discours» selon Charolles. Quand les AdvCA ont une PCP, nous nous attendons à trouver un ou plusieurs indicateurs contextuels d'un autre cadre ou d'une autre perspective dans les énoncés qui précèdent l'énoncé marqué par l'AdvCA. Pour tester cette hypothèse, nous analysons le contexte discursif de 50 occurrences de l'AdvCA en pratique qui, contrairement à en réalité et en fait, a toujours une PCP.

Notre repérage manuel prend en compte toutes les formes qui peuvent être considérées comme indicateurs contextuels d'un changement de perspective au niveau du contenu et qui apparaissent dans le contexte discursif gauche de l'AdvCA en pratique. La fenêtre d'analyse est limitée à environ 60 mots par le concordancier implémenté sur la plateforme BTLC.Primestat. Cette fenêtre couvre entre un et trois énoncés précédant l'AdvCA en pratique pour que l'on puisse garantir un lien argumentatif direct entre ces énoncés et l'énoncé introduit par l'AdvCA. En d'autres termes, ceci nous permet de ne tenir compte que des cas où les indicateurs contextuels et l'AdvCA appartiennent à une même séquence de mouvements discursifs.

Dans un échantillon aléatoire de 50 occurrences de en pratique tirées du corpus CoVaNa-FR, nous avons trouvé une large gamme d'indicateurs contextuels introduisant un cadre ou une perspective qui sont différents de ceux introduits par l'AdvCA. Parmi les indicateurs contextuels repérés nous trouvons:

1. des formes qui mentionnent un énonciateur différent et des formes évidentielles (l'association Omega-Yoga, M. Bertrand, selon lui, l'arrêt a confirmé que...) (12 occurrences); 
2. des verbes de positionnement et d'état (point de vue/opinion) indiquant une certaine incertitude par rapport à ce qui est dit et ainsi introduisant une perspective faisant référence aux «apparences» ou à la «théorie» (trouver, indiquer, affirmer, dire, penser, décrire, déclarer, proposer, croire, se proposer, être sûr...) (14 occurrences);

3. des formes cadratives indiquant un cadre de la «théorie» ou des «apparences» opposé au cadre introduit par en pratique (la théorie, en théorie, en principe, théoriquement, en apparence...) (11 occurrences);

4. des formes indiquant tout type de cadre thématique opposé à/différent de en pratique (de façon purement symbolique, idéalement, en termes d'audience, dans l'esprit de...) (10 occurrences);

5. des verbes au conditionnel/à l'imparfait exprimant une incertitude par rapport au contenu introduit (5 occurrences).

Dans notre échantillon, pour 8 des 50 occurrences de en pratique, nous ne trouvons pas ce type d'introducteurs contextuels qui incontestablement marqueraient un cadre différent dans le contexte discursif gauche de l'AdvCA. Néanmoins, au vu de la présence d'indications plus implicites dans le contexte, nous considérons intuitivement que l'AdvCA a une PCP aussi dans tous ces cas sans marquage explicite d'une perspective différente, par exemple quand intervient un cadre temporel qui crée néanmoins un certain contraste:

[14] En 2006, les exportations de fictions, d'animations et de documentaires ont connu une hausse de 4,2 \%, pour atteindre 157 millions d'euros. En pratique, même si elles restent encore modestes, les ventes de programmes français connaissent un regain qui ne dément pas depuis trois ans.

Pour 42 occurrences de en pratique, nous trouvons un ou même plusieurs indicateurs contextuels signalant une perspective différente adoptée dans les énoncés qui précèdent en pratique (voir la liste non exhaustive ci-dessus), ce qui indique que l'AdvCA marque un changement de perspective au niveau du contenu. Ainsi, des formes évidentielles et les verbes indiquant une incertitude par rapport au contenu présenté, notamment, se combinent pour introduire une perspective différente. Notre objectif n'est pas de proposer une classification précise de tous les introducteurs, en dépit de l'intérêt qu'une telle étude peut présenter, mais de déterminer si l'on en trouve dans la plupart des cas dans le contexte gauche de en pratique. L'échantillon analysé semble confirmer cette hypothèse et ainsi la fiabilité du codage des emplois à PCP. La cooccurrence d'un ou de plusieurs de ces indicateurs contextuels avec l'AdvCA s'avère être un indice d'un changement de perspective au niveau du contenu introduit dans la suite du discours par cet AdvCA avec une PCP. Nous testons ce constat dans les analyses manuelles suivantes pour les trois AdvCA en pratique, en réalité et en fait et dans la section 7 au moyen d'un calcul de la spécificité de ces indicateurs contextuels en tant que cooccurrents des AdvCA. 


\section{Répartition des différents emplois des adverbiaux cadratifs abstraits}

Le codage proposé s'avère fiable pour identifier des formes qui ont des emplois à PCP: le codage de 50 occurrences de en pratique a montré que, pour presque tous ses emplois à PCP, on trouve des indicateurs contextuels. Pour appliquer le codage de la PCP et pour déterminer la répartition des différents emplois, il est nécessaire de trier manuellement des échantillons non seulement pour en pratique, mais pour les trois AdvCA. Nous procédons ainsi à ce tri manuel également pour en réalité et en fait. Nous avons l'hypothèse que, comme les autres formes cadratives, en réalité et en fait devraient avoir des emplois à PCP où ce type d'indicateurs contextuels intervient dans leur contexte discursif gauche. Mais nous supposons également que en fait et en réalité peuvent avoir une $\mathrm{PCE}$. Dans ce cas, nous nous attendons à ne trouver aucun des introducteurs signalant une perspective différente au niveau du contenu dans le contexte gauche des AdvCA. Nous appliquons le même codage manuel déjà testé pour en pratique à des échantillons aléatoires de 50 occurrences de en réalité et en fait afin de vérifier la pertinence de ce codage pour la distinction entre PCP et PCE. En outre, nous cherchons à quantifier la répartition des emplois à PCP par rapport aux emplois à PCE en comparant leurs fréquences dans les échantillons analysés (tableau 2).

\begin{tabular}{|l|r|l|l|l|}
\hline \multirow{2}{*}{} & \multicolumn{3}{|l|}{\begin{tabular}{l}
\multicolumn{2}{l|}{ Portée cadrative propositionnelle } \\
(PCP)
\end{tabular}} & \multicolumn{2}{|l|}{ Portée cadrative énonciative (PCE) } \\
\cline { 2 - 6 } & Fréquence & $\begin{array}{l}\text { Pourcentage de } \\
\text { l'emploi }\end{array}$ & Fréquence & \multicolumn{2}{l|}{$\begin{array}{l}\text { Pourcentage de } \\
\text { l'emploi }\end{array}$} \\
\hline en réalité & 31 & $62 \%$ & 19 & $38 \%$ \\
\hline en fait & 25 & $50 \%$ & 25 & $50 \%$ \\
\hline
\end{tabular}

Tableau 2 - Répartition des emplois à $\mathrm{PCP}$ et à $\mathrm{PCE}$ de en réalité et en fait (fréquences et pourcentages pour 50 occurrences aléatoires analysées)

Le codage des emplois à PCP en fonction de la présence d'indicateurs contextuels fait ressortir une différence importante entre les trois AdvCA analysés. En pratique n'a que des emplois à $\mathrm{PCP}$, tandis que en réalité et en fait ont des emplois à $\mathrm{PCP}$ et des emplois à PCE. Pour en réalité, les emplois à PCP restent plus fréquents que les emplois à PCE (38/62\%). Pour en fait, les emplois à PCP et à PCE représentent chacun la moitié de l'échantillon $(50 / 50 \%)$.

Lors du tri manuel, un certain nombre d'emplois s'avèrent être intraprédicatifs malgré la requête que nous avons formulée à cet égard (partie 4). Il y a 15 \% d'erreurs pour les occurrences de en fait (par exemple Royal en fait le thème central de son intervention), et $4 \%$ pour les occurrences de en réalité (par exemple transformer leurs promesses en réalité). Suite à ce constat, selon lequel le paramétrage de la requête (exclusion des formes verbales de faire par la recherche de fait comme «nom» et 
exclusion de tous les verbes précédant les AdvCA pour éliminer le plus grand nombre possible d'emplois intraprédicatifs) a échoué dans un certain nombre de cas pour en réalité et en fait, nous avons dû élargir chacun des échantillons afin d'obtenir 50 occurrences extraprédicatives (PCP/PCE) des AdvCA.

Les exemples suivants servent à démontrer comment a été appliqué notre codage de la PCP en fonction de la présence d'introducteurs contextuels (soulignés) signalant une perspective différente de celle introduite par l'AdvCA au niveau du contenu.

\subsection{Portée cadrative propositionnelle (PCP)}

[15] "Après quinze ans à Zurich, j'ai un peu l'impression d'en avoir fait le tour», soupire Michael. En réalité, l'enfant des montagnes saint-galloises n'envisage pas une seconde de quitter sa ville d'adoption.

[16] Depuis le retour à la paix, en 1995, les forces de police sont placées sous la responsabilité des deux «entités» qui constituent la Bosnie, théoriquement unie mais $e n$ réalité toujours divisé $[s i c]$ : la Republika Srpska et la Fédération croato-musulmane.

[17] [...] la répartition de leurs rôles respectifs, telle qu'elle est prévue dans le texte de la Constitution, ne correspond pas à la réalité: l'article 20 prévoit que «le gouvernement détermine et conduit la politique de la nation", alors qu'en fait c'est le président qui la détermine, mais c'est le gouvernement qui en est responsable devant le Parlement, alors que le président, lui, ne l'est pas.

[18] À Stockholm, on appelle cela la «semaine des Nobel». En fait, plutôt dix jours pour les lauréats, qui enchaînent les réceptions officielles, concerts et autres visites dans les écoles.

Dans ces quatre exemples tirés des échantillons, nous identifions des indicateurs contextuels d'une perspective différente au niveau du contenu dans le contexte discursif gauche de en réalité ou de en fait (impression; théoriquement; À Stockholm, on appelle cela). L'exemple [17] contient même plusieurs indicateurs contextuels. Le changement de perspective indiqué par une opposition entre le cadre qui appartient aux «apparences» (prévoit) et le cadre des «faits» (en fait) est déjà marqué dans la phrase précédente (prévue/ne correspond pas à la réalitê).

Dans les exemples analysés, il y a aussi un grand nombre d'emplois à PCE (38\% pour en réalité et $50 \%$ pour en fait). Contrairement aux exemples précédents, les occurrences [19] à [22] n'ont pas d'indicateurs contextuels dans leur contexte discursif gauche.

\subsection{Portée cadrative énonciative (PCE)}

[19] «Le problème, poursuit M. Milot, c'est que souvent, aussitôt qu'il est question de religion au Québec, certains disjonctent! En réalité, certains ont des comptes 
à régler avec la religion et ils sont incapables de faire la différence entre leurs états d'âme personnels et ce que dit la loi. [...]»

[20] Un seul type d'accessoire est mis à profit: une flopée de quilles scintillantes que plusieurs jongleurs s'échangent en les faisant virevolter de toutes les manières: à l'aveugle, en mouvement, à reculons, etc. Mais en réalité, chaque artiste qui prend part à ce spectacle a de quoi amuser et fasciner avec ses performances spectaculaires [...].

[21] Rien n'est plus pareil. Au coin de ma rue, le magasin de surplus de l'armée a écoulé tous ses masques à gaz et combinaisons doublées à l'épreuve de l'anthrax, de la variole et des vapeurs de chlorobenladène. On n'avait pas vu ça depuis... depuis le Sommet des Amériques de Québec, en fait.

[22] C'est muni de cette prudence focale que Frédéric Lecloux s'est engagé sur le chemin désormais culte de L'Usage du monde de Nicolas Bouvier. Mais ce jeune photographe né à Bruxelles et établi dans la Drôme avait une autre raison, bien plus intéressante en fait, de garder ses distances avec son livre fétiche.

En réalité dans les exemples [19] et [20] et en fait dans [22] introduisent un énoncé qui est mis à l'avant-plan discursif par le locuteur. Dans l'exemple [19], l'AdvCA introduit clairement une reformulation de l'énoncé précédent en ajoutant une précision du contenu (Rossari, 1992: 153). Dans l'exemple [20], en réalité souligne le fait que l'énoncé introduit par le connecteur mais et par l'AdvCA est mis en avant dans le discours par rapport au premier énoncé dans la structure concessive (voir Rossari [2015] pour une description de la fonction discursive du connecteur mais). Dans l'exemple [22], en fait fait partie d'une incise insérée par le locuteur ayant une fonction de commentaire explicatif supplémentaire dans son récit, qui, autrement, raconterait l'histoire du jeune photographe d'une manière plus neutre.

L'exemple [21] est en quelque sorte spécial, car en fait semble être un marqueur discursif dont la portée cadrative parait plus ou moins occultée. Nous voudrions saisir cette occasion pour souligner que notre codage ne permet qu'une distinction entre la PCP et la PCE des AdvCA. Il n'est en revanche pas possible de distinguer entre différents emplois à PCE (emploi comme connecteur reformulatif ou emploi qui s'approche encore plus de celui d'un marqueur discursif - voir Blumenthal [1996]).

En résumé, le codage en fonction de la présence d'indicateurs contextuels d'une perspective différente concernant le contenu propositionnel permet d'identifier les emplois à PCP. Les AdvCA en pratique, en réalité et en fait ont en commun l'emploi à $\mathrm{PCP}(100 \%, 62 \%, 50 \%)$. En réalité et en fait ont aussi un nombre important d'emplois à PCE (38\%, $50 \%)$. Ces AdvCA marquent un changement de perspective au niveau énonciatif en indiquant le positionnement des énoncés dans le discours par le locuteur. Dans ce cas, on ne trouve pas d'indicateurs contextuels dans leur contexte discursif gauche. 


\section{Cooccurrents spécifiques des adverbiaux cadratifs abstraits}

Partant des résultats du repérage préliminaire des indicateurs contextuels qui introduisent une autre perspective (ou un autre cadre) dans le contexte gauche des AdvCA en pratique, en réalité et en fait, nous passons à un repérage automatique sur corpus des cooccurrents spécifiques gauches de ces trois AdvCA. Notre but est de vérifier dans une perspective statistique si ce genre d'introducteurs contextuels intervient spécifiquement en cooccurrence avec tous les AdvCA qui peuvent avoir une PCP. Rappelons-nous qu'avec en pratique, qui a toujours une PCP, nous avons presque systématiquement trouvé des éléments dans le contexte discursif gauche de l'AdvCA. Ceci indiquait clairement une perspective différente au niveau du contenu. Nous avons trouvé une situation similaire pour en fait et en réalité dans les emplois où ces formes ont une PCP. Nous cherchons donc ces types d'indicateurs contextuels parmi les cooccurrents spécifiques des trois AdvCA. Ce repérage ne distingue pas entre PCP et PCE, mais il nous permet d'évaluer la fiabilité du codage proposé pour des AdvCA qui ont régulièrement une PCP. L'analyse sur corpus traite un échantillon aléatoire d'occurrences qui contient des exemples d'emplois à PCP et à PCE mélangés. Nous devrions trouver dans la liste des cooccurrents spécifiques des AdvCA des indicateurs contextuels que nous avons déjà repérés manuellement en fonction du codage proposé ou encore d'autres éléments similaires. Ceci indiquerait que les trois AdvCA non seulement partagent dans certains cas le même type de contexte discursif gauche dans lequel une perspective différente au niveau du contenu est introduite, mais aussi que la cooccurrence des AdvCA avec ces introducteurs contextuels est statistiquement spécifique.

Alors que le repérage manuel des indicateurs contextuels permet de coder les occurrences des AdvCA en fonction du mouvement discursif dans lequel ils sont utilisés, il ne permet pas de se prononcer sur la spécificité des cooccurrences des AdvCA avec ces indicateurs. Nous cherchons à savoir si des indicateurs contextuels sont typiquement utilisés avec tous les AdvCA qui peuvent avoir une PCP. Si c'est bien le cas, ce serait un autre indice de la pertinence de notre codage. Le repérage automatique des cooccurrents spécifiques est effectué sur la plateforme BTLC.Primestat qui applique un calcul de log-likelibood. Cette mesure d'association statistique permet d'indiquer quels cooccurrents d'un AdvCA sont «plus fréquents dans l'environnement de ce mot qu'on n'aurait pu le prédire sur la base des fréquences absolues des mots en question» (Blumenthal, 2008: 38). Le calcul est une indication de l'évidence statistique (evidence-based measures [Evert, 2008]) contre l'hypothèse nulle de la distribution aléatoire du mot pivot et du mot cooccurrent dans un corpus. Le calcul compare le nombre de cooccurrences avec une estimation de cette fréquence dans le cas hypothétique où les deux éléments seraient distribués de manière aléatoire dans le corpus. Si l'écart entre la fréquence de cooccurrence observée et estimée est statistiquement significatif, la cooccurrence est traitée comme étant spécifique. Dans ce cas, on peut l'interpréter comme un indice d'une association typique et régulière de l'AdvCA et de l'indicateur contextuel. 
Pour la recherche des cooccurrents spécifiques des AdvCA, nous avons appliqué un seuil de pertinence pour le log-likelibood fixé à 10,83. Ceci représente une valeur $p<0,001$ et une probabilité de plus de $99,9 \%$ que l'hypothèse nulle de la distribution aléatoire des éléments soit à rejeter ${ }^{5}$. Au-dessus de ce seuil, les valeurs indiquent donc une cooccurrence spécifique et non due au hasard. Le repérage des cooccurrents spécifiques d'un pivot (ici l'AdvCA) nécessite la définition d'un empan de recherche dans lequel les cooccurrents sont cherchés. Nous avons choisi un empan de 15 mots à gauche du mot pivot afin de couvrir au moins l'énoncé précédant l'AdvCA dans son ensemble et au maximum deux à trois énoncés courts ${ }^{6}$. Le paramétrage de la requête choisi pour les cooccurrents permet de chercher des syntagmes formés par un marqueur prédéterminant un lemme (sur la base de l'annotation du corpus pour les relations syntaxiques [Diwersy, 2014: 49-51]). Ainsi, il est possible de trouver un ensemble "préposition + lemme» en tant que cooccurrent d'un AdvCA (par exemple en théorie comme cooccurrent complexe et non seulement théorie).

Pour les sections suisse, française et canadienne du CoVaNa-FR avec 134939662 mots, les résultats du repérage des cooccurrents spécifiques révèlent que les trois AdvCA ont plusieurs cooccurrents spécifiques intervenant dans leur contexte discursif gauche qui peuvent introduire une perspective différente de celle introduite par l'AdvCA au niveau du contenu (tableau 3).

Dans la liste des cooccurrents spécifiques des AdvCA qui ont tous des emplois à $\mathrm{PCP}$ (seule portée cadrative possible pour en pratique, l'une des deux portées cadratives possibles pour en réalité et en fait), nous trouvons plusieurs indicateurs contextuels d'une perspective différente au niveau du contenu. Les indicateurs contextuels varient selon l'AdvCA. En pratique, qui est aussi l'AdvCA le moins fréquent dans notre corpus, a deux cooccurrents spécifiques qui sont aussi des AdvCA : en théorie et en principe. À ces adverbiaux s'ajoutent des adverbes cadratifs (théoriquement, formellement) et des formes potentiellement cadratives faisant référence à la "théorie» (pour théorie, théorique) ou au "principe» (principe). En fait a comme cooccurrents spécifiques six verbes qui indiquent une perspective (dire, sembler, croire, penser, parler, se tromper) et un adverbe cadratif qui fait référence au cadre des «apparences» (apparemment). En réalité a les cooccurrents spécifiques les plus diversifiés. On y trouve deux adverbes cadratifs (officiellement, apparemment), des verbes et adjectifs indiquant une perspective ou un cadre qui appartiennent

5. Pour les aspects plus techniques des mesures d'association utilisées dans l'analyse sur corpus des cooccurrences d'items linguistiques, voir par exemple les travaux de Evert (2008), Wiechmann (2008) et Gries (2010).

6. Pour une grande majorité des cas, cet empan couvre les deux mouvements discursifs dont font partie l'énoncé introduit par l'AdvCA et l'énoncé précédent dans lequel paraît le cooccurrent. Nous avons mené des tests qui ont montré que des empans de 5 et de 7 mots sont trop peu étendus, car ils ne couvrent pas au moins un énoncé complet. De même, des empans de 20 et de 25 mots sont trop larges et incluent d'habitude trop de bruit (par exemple des énoncés qui n'ont pas de liens directs avec l'énoncé introduit par l'AdvCA). Nous n'avons pas pu prendre en compte comme critère l'unité paragraphe: en introduisant un paragraphe, l'adverbial peut avoir une portée différente et plus large. 


\begin{tabular}{|c|c|c|c|c|c|}
\hline en fait & $\begin{array}{l}\text { Fréquence de } \\
\text { cooccurrence }\end{array}$ & $\begin{array}{l}\text { Fréquence } \\
\text { pivot }\end{array}$ & $\begin{array}{l}\text { Fréquence } \\
\text { cooccurrent }\end{array}$ & Log-likelibood & $\begin{array}{l}\text { Rang } \\
\text { log-likelibood }\end{array}$ \\
\hline dire & 215 & 7531 & 124056 & 90,69 & 5 \\
\hline sembler & 68 & 7531 & 37678 & 31,56 & 28 \\
\hline croire & 59 & 7531 & 31117 & 30,57 & 29 \\
\hline penser & 57 & 7531 & 31466 & 26,68 & 33 \\
\hline parler & 57 & 7531 & 31515 & 26,58 & 34 \\
\hline se tromper & 10 & 7531 & 1706 & 21,78 & 49 \\
\hline apparemment & 9 & 7531 & 2569 & 12,07 & 113 \\
\hline \multicolumn{6}{|l|}{ en réalité } \\
\hline officiellement & 29 & 2468 & 6141 & 110,45 & 1 \\
\hline croire & 37 & 2468 & 31117 & 51,62 & 2 \\
\hline en apparence & 9 & 2468 & 757 & 50,27 & 3 \\
\hline en théorie & 8 & 2468 & 743 & 43,13 & 8 \\
\hline de prétendre & 5 & 2468 & 311 & 31,79 & 13 \\
\hline paraître & 18 & 2468 & 14459 & 26,39 & 17 \\
\hline sembler & 30 & 2468 & 37678 & 24,61 & 19 \\
\hline prétendu & 5 & 2468 & 926 & 20,31 & 23 \\
\hline dire & 61 & 2468 & 124056 & 17,27 & 29 \\
\hline apparemment & 6 & 2468 & 2569 & 15,11 & 38 \\
\hline illusion & 5 & 2468 & 1920 & 13,56 & 41 \\
\hline assurer & 19 & 2468 & 26063 & 13,44 & 42 \\
\hline \multicolumn{6}{|l|}{ en pratique } \\
\hline en théorie & 58 & 1209 & 1294 & 690,15 & 1 \\
\hline pour théorie & 7 & 1209 & 58 & 97,17 & 4 \\
\hline théoriquement & 8 & 1209 & 1937 & 57,15 & 7 \\
\hline théorique & 9 & 1209 & 3884 & 54,04 & 8 \\
\hline en principe & 9 & 1209 & 6161 & 45,93 & 10 \\
\hline formellement & 5 & 1209 & 3438 & 25,47 & 17 \\
\hline principe & 8 & 1209 & 20551 & 20,97 & 24 \\
\hline
\end{tabular}

Tableau 3 - Cooccurrents spécifiques de chacun des AdvCA ayant une fonction d'indicateur contextuel d'une perspective différente au niveau du contenu de celle introduite par l'AdvCA 
aux «apparences» (croire, de prétendre, prétendu, paraître, sembler, dire, assurer), un substantif indiquant potentiellement un cadre opposé à la «réalité» (illusion) et l'AdvCA en apparence. Les résultats confirment l'hypothèse selon laquelle les AdvCA en pratique, en réalité et en fait se combinent typiquement avec des indicateurs contextuels signalant une perspective différente de celle introduite par l'AdvCA au niveau du contenu.

Pourtant, la recherche des cooccurrents spécifiques ne répond pas à la question concernant la possibilité qu'un tel indicateur soit systématiquement présent dans le contexte gauche des AdvCA lorsqu'ils ont une PCP. De même, ce type d'analyse ne fait aucune mention de la répartition des emplois à PCP et à PCE des AdvCA en réalité et en fait. Mais le fait qu'on trouve ce type d'indicateurs contextuels en tant que cooccurrents spécifiques des AdvCA dans leur contexte discursif gauche confirme notre hypothèse qu'il existe des liens importants entre ces indicateurs contextuels et les trois AdvCA dont il est question ici. Ce que l'analyse cooccurrentielle ne peut pas indiquer a en fait été confirmé par nos analyses manuelles des occurrences des AdvCA: dans les emplois à PCP, les trois AdvCA sont accompagnés d'introducteurs contextuels; dans les emplois à $\mathrm{PCE}$ de en réalité et en fait, ce n'est pas le cas. La répartition des emplois à PCP et à PCE peut être cernée par un tri manuel au moyen du codage qui ainsi s'avère opérationnel.

\section{Dans la pratique, dans la réalité et dans les faits}

Le codage des emplois à $\mathrm{PCP}$ a été testé pour trois $\mathrm{AdvCA}$ (en réalité, en fait, en pratique) seulement, dont l'un d'entre eux (en pratique) n'a que des emplois à PCP et les deux autres (en réalité, en fait) ont également des emplois à PCE. Nous élargissons notre analyse en ajoutant trois autres AdvCA qui, comme en pratique, ont uniquement des emplois à $\mathrm{PCP}$ : dans la pratique, dans la réalité et dans les faits. Ces AdvCA sont formés avec la préposition dans qui participerait «le plus souvent à la construction du sens référentiel des énoncés» (Vigier, $2015: 245)$. Nous avons brièvement indiqué dans la partie 3 que en réalité, en fait et en pratique peuvent dans certains cas être paraphrasés par l'une de ces formes référentielles.

Un repérage des cooccurrents spécifiques en fonction des mêmes critères déjà esquissés ci-dessus fait sortir la liste de cooccurrents spécifiques présentée dans le tableau 4.

On constate que, par rapport à en pratique, en réalité et en fait, ces trois AdvCA ont moins d'introducteurs contextuels comme cooccurrents spécifiques, ce qui est en partie dû au fait qu'ils sont nettement moins fréquents dans le corpus. Le codage manuel de 50 occurrences aléatoires pour chacun des trois AdvCA démontre un emploi similaire à celui de en pratique:

- 41 occurrences sur 50 de dans la pratique ont au moins un indicateur contextuel dans leur contexte discursif gauche (9 occurrences sans indicateur explicite, dont une dans une construction intraprédicative : vont entrer immédiatement dans la pratique); 
- 45 occurrences sur 50 de dans la réalité ont au moins un indicateur contextuel dans leur contexte discursif gauche (5 occurrences sans indicateur explicite);

- 44 occurrences sur 50 de dans les faits ont au moins un indicateur contextuel dans leur contexte discursif gauche (6 occurrences sans indicateur explicite).

Nous en concluons que dans la pratique, dans la réalité et dans les faits partagent un emploi à PCP similaire à celui des autres AdvCA analysés. Toutes ces formes portent sur le contenu des énoncés et marquent un changement de perspective au niveau du contenu quand elles interviennent dans la même séquence de mouvements discursifs que des indicateurs contextuels signalant une autre perspective ou un autre cadre. Les AdvCA qui peuvent avoir une PCE (en réalité, en fait) et les AdvCA référentiels (dans la pratique, dans la réalité, dans les faits) ainsi que en pratique ont tous plusieurs cooccurrents spécifiques qui sont introducteurs contextuels d'une perspective différente au niveau du contenu propositionnel. En revanche, ces adverbiaux, pour la plupart, ne partagent pas les formes exactes (sauf par exemple en théorie). Dans un futur travail, il serait intéressant de comparer les listes de cooccurrents spécifiques des AdvCA de manière plus détaillée. Les cooccurrents spécifiques qui sont similaires pour tous les AdvCA paraissent fortement associés à l'emploi à PCP. Mais en réalité et en fait partagent-ils d'autres cooccurrents spécifiques qui seraient plus associés aux emplois à PCE, permettant ainsi un codage de la PCE?

\begin{tabular}{|c|c|c|c|c|c|}
\hline $\begin{array}{l}\text { dans la } \\
\text { pratique }\end{array}$ & $\begin{array}{l}\text { Fréquence de } \\
\text { cooccurrence }\end{array}$ & $\begin{array}{l}\text { Fréquence } \\
\text { pivot }\end{array}$ & $\begin{array}{l}\text { Fréquence } \\
\text { cooccurrent }\end{array}$ & Log-likelihood & $\begin{array}{l}\text { Rang } \\
\text { log-likelibood }\end{array}$ \\
\hline en théorie & 11 & 382 & 743 & 106,88 & 1 \\
\hline pour théorie & 7 & 382 & 46 & 100,66 & 2 \\
\hline \multicolumn{6}{|l|}{ dans la réalité } \\
\hline sur (le) papier & 9 & 449 & 958 & 68,97 & 3 \\
\hline sembler & 8 & 449 & 37678 & 10,93 & 20 \\
\hline \multicolumn{6}{|l|}{ dans les faits } \\
\hline sur (le) papier & 9 & 1120 & 958 & 52,72 & 4 \\
\hline officiellement & 13 & 1120 & 6141 & 49,21 & 5 \\
\hline en théorie & 6 & 1120 & 743 & 38,26 & 8 \\
\hline
\end{tabular}

Tableau 4 - Cooccurrents spécifiques de chacun des adverbiaux cadratifs ayant une fonction d'introducteur d'une perspective différente au niveau du contenu de celle introduite par l'adverbial 


\section{Conclusion}

Nous aimerions mettre en avant quelques aspects relevés par notre étude sur corpus. Dans une perspective théorique, le modèle triadique permet de représenter la polyvalence des sens des AdvCA d'une manière systématique et en fonction d'un seul critère: la cible de la portée cadrative. Nous avons montré qu'une distinction entre les différents emplois des AdvCA est possible en fonction du type de portée cadrative (PCP ou PCE). L'application pratique de ces réflexions théoriques au moyen d'une étude sur corpus a confirmé qu'un codage manuel en fonction de la présence d'indicateurs contextuels d'une perspective différente de celle introduite par l'AdvCA au niveau du contenu peut être fiable. La pertinence de ce codage a été assurée par un repérage automatique des cooccurrents spécifiques, qui a fait relever que des formes introduisant une perspective ou un cadre qui diffèrent de celui introduit par les AdvCA interviennent spécifiquement dans le contexte discursif gauche des AdvCA. Ainsi, nous avons montré qu'une large gamme de ces types d'indicateurs contextuels doit être prise en compte afin de pouvoir distinguer les emplois à PCP et ceux à PCE. Il serait intéressant d'analyser plus en détail ce paradigme de formes dans une future étude, afin d'affiner le codage proposé pour les emplois à PCP. De même, comme nous l'avons indiqué, il serait intéressant de déterminer si les AdvCA à PCE partagent également certains types de cooccurrents. Si c'est le cas, il serait possible d'établir un codage similaire et opérationnel pour les emplois à PCE. Nous pensons, par exemple, à des mouvements discursifs où un connecteur (tel que mais), qui met en contraste plusieurs énonciations dans un discours (Rossari, 2015), intervient avec un AdvCA.

Notre étude sur corpus a fait ressortir que l'AdvCA en pratique (comme dans la pratique, dans la réalité et dans les faits) n'a qu'un emploi à PCP, alors que les $\mathrm{AdvCA}$ en réalité et en fait ont des emplois à $\mathrm{PCP}$, mais également des emplois à PCE aussi fréquents. On pourrait se demander si la répartition des différents emplois est caractéristique du genre discursif journalistique. Nous imaginons que des genres plus «informatifs», qui n'évaluent pas explicitement des opinions ou perspectives (encyclopédies, textes scientifiques, etc.), contiennent surtout des formes à PCP qui structurent l'information, et moins de formes à PCE, alors que des genres «argumentatifs» plutôt oraux (discours politiques, conversations orales, échanges en ligne, etc.) ont besoin et de la PCE pour indiquer la pertinence d'un argument et de la PCP pour la structuration des blocs d'information.

Lors du codage manuel, nous avons remarqué que, dans un grand nombre d'emplois, les AdvCA à PCP servent à contraster deux perspectives de deux énonciateurs bien différents : les indicateurs contextuels introduisent souvent une citation directe ou indirecte des propos d'une tierce personne (voir Schrepfer-André [2005] pour une piste intéressante concernant l'emploi de la forme évidentielle selon $X$ ), tandis que les AdvCA en pratique, en réalité et en fait introduisent la perspective de l'article du journal. Il en va de même pour dans la pratique, dans la réalité et dans les faits. Une comparaison avec ces AdvCA référentiels a confirmé la fiabilité 
du codage manuel des emplois à $\mathrm{PCP}$ et a montré que ces formes, tout comme en pratique, n'ont que des emplois à $\mathrm{PCP}$.

Enfin, il serait souhaitable que le codage proposé puisse être appliqué systématiquement à d'autres formes cadratives afin de mieux cerner le contexte discursif dans lequel elles interviennent de manière typique. Nous espérons ainsi obtenir de nouvelles pistes sur les différentes fonctions et portées des formes cadratives. Le grand défi de ce type d'études sur corpus est le fait que les relations discursives ne sont pas ou peu annotées dans les corpus. Cette contrainte peut être contournée par une adaptation des analyses cooccurrentielles traditionnelles. Notre étude sur corpus montre qu'il est possible de chercher d'autres marqueurs avec une fonction discursive en tant que cooccurrents spécifiques d'une forme donnée afin de déterminer les types de mouvements discursifs dans lesquels cette forme est typiquement utilisée. Les liens entre mouvements discursifs, marqueurs représentant ces mouvements discursifs et forme en question sont des indices importants pour la description linguistique des différentes fonctions que cette forme peut remplir dans différents contextes discursifs.

\section{Références}

Blumenthal, P. 1996. Le connecteur en fait. In C. Muller (éd.), Dépendance et intégration syntaxique: subordination, coordination, connexion. Tübingen: Niemeyer: 257-269.

Blumenthal, P. 2008. Combinatoire des prépositions: approche quantitative. Langue française 157: 37-51.

Blumenthal, P. 2017. Évolution de la combinatoire prépositionnelle: le cas de en. In C. Badiou-Monferran, S. Bajrić et P. Monneret (éd.), Penser la langue: sens, texte, bistoire. Hommages à Olivier Soutet. Paris: H. Champion: 161-176.

Charolles, M. 1995. Cohésion, cohérence et pertinence du discours. Travaux de linguistique 29: $125-151$.

Charolles, M. 1997. L'encadrement du discours: univers, champs, domaines et espaces. Cabier de recherche linguistique $6: 1-73$. Une version en ligne est accessible à l'adresse suivante : http://www.lattice.cnrs.fr/IMG/pdf/cadresdisc.pdf.

Charolles, M. et Pietrandrea, P. 2012. En réalité: de la modalisation à l'organisation du discours. Travaux de linguistique 64: 111-142.

Charolles, M. et Vigier, D. 2005. Les adverbiaux en position préverbale: portée cadrative et organisation des discours. Langue française 148: 9-30.

Combettes, B. 2005. Les constructions détachées comme cadres de discours. Langue française 148: 31-44.

De Mulder, W. et Амiot, D. 2013. En: de la préposition à la construction. Langue française 178: 21-39.

Ducrot, O. 1980. Analyses pragmatiques. Communications 32:11-60.

Evert, S. 2008. Corpora and Collocations. In A. Lüdeling et M. Kytö (éd.), Corpus linguistics. An International Handbook. Berlin: De Gruyter: 1212-1248. 
FAGARD, B. et Combettes, B. 2013. De en à dans, un simple remplacement? Une étude diachronique. Langue française 178: 93-115.

Ferret, O., Grau, B., Minel, J.-L. et Porhiel, S. 2001. Repérage de structures thématiques dans des textes. In TALN - RECITAL 2001: $8^{e}$ conférence annuelle sur le Traitement automatique des langues naturelles (2-5 juillet 2001, Tours). Paris: ATALA (Association pour le Traitement automatique des langues): 163-172. En ligne à l'adresse suivante: http://www.atala.org/content/taln-2001.

Gries, S. T. 2010. Useful Statistics for Corpus Linguistics. In A. SÁNChez Pérez et M. Almela Sánchez (éd.), A Mosaic of Corpus Linguistics: Selected Approaches. Francfortsur-le-Main - Berlin - Berne: P. Lang: 269-291.

Guimier, C. 1996. Les adverbes du français: le cas des adverbes en -ment. Gap - Paris: Ophrys.

Guimier, C. 2007. Adverbe de domaine et structuration du discours. In A. CELle, S. Gresset et R. Huart (éd.), Les connecteurs, jalons du discours. Berne: P. Lang: 43-70.

Gülich, E. et КотsсHI, T. 1983. Les marqueurs de la reformulation paraphrastique. Cabiers de linguistique française 5 : 305-346. En ligne à l'adresse suivante: http://clf.unige.ch/ index.php/download_file/view/410/159/.

IORDANSKAJA, L. et MeL'čUK, I. 1995. Traitement lexicographique de deux connecteurs textuels du français contemporain. In H. BAT-ZeEv Shyldkrot et L. Kupferman (éd.), Tendances récentes en linguistique française et générale: volume dédié à David Gaatone. Amsterdam - Philadelphie: J. Benjamins: 211-236.

Porhiel, S. 2005. Les séquences thématiques. Langue française 148: 111-126.

Prévost, S. 2003. Les compléments spatiaux: du topique au focus en passant par les cadres. Travaux de linguistique 47 : 51-77.

Rossari, C. 1992. De fait, en fait, en réalité: trois marqueurs aux emplois inclusifs. Verbum 3: 139-161.

RossARI, C. 1997. Les opérations de reformulation: analyse du processus et des marques dans une perspective contrastive français-italien. Berne - Berlin - Paris: P. Lang.

Rossari, C. 2015. Une concession implique-t-elle une opposition? In A. FERrari, L. LALA et R. Stojmenova (éd.), Testualità: fondamenti, unità, relazioni. Atti del convegno internazionale di studi Linguistica testuale: «Teorie, metodi, fenomeni, strutture» (Basilea, 2 luglio-4 luglio 2014). Florence: Cesati Editore: 189-203.

Rossari, C. 2016. Les fluctuations de sens dans quelques formes modales à la lumière d'une approche quantitative et qualitative. Studii de lingvistică $6: 127-142$.

Roulet, E. 1987. Complétude interactive et connecteurs reformulatifs. Cabiers de linguistique française 8 : 111-140. En ligne à l'adresse suivante: http://clf.unige.ch/index.php/ download_file/view/374/156/.

SChrepfer-ANDrÉ, G. 2005. Incidence des formes de reprise du SN régime des SP en selon X énonciatifs sur leur portée phrastique et textuelle. Langue française 148: 80-94.

Vigier, D. 2003. Les syntagmes prépositionnels en «en N» détachés en tête de phrase référant à des domaines d'activité. Lingvistica Investigationes 26 (1) : 97-122.

VIGIER, D. 2015. Les prépositions en, dans et dedans au XVI ${ }^{\mathrm{e}}$ siècle. Approche statistique et combinatoire. Le français moderne 2: 230-247. 
Wiechmann, D. 2008. On the Computation of Collostruction Strength: Testing Measures of Association as Expressions of Lexical Bias. Corpus Linguistics and Linguistic Theory 4 (2) : 253-290.

\section{Corpus}

Diwersy, S. 2013. BTLC.Primestat. En ligne à l'adresse suivante: http://persan.rom. uni-koeln.de/btlsc/.

Diwersy, S. 2014. The Varitext Platform and the Corpus des Variétés Nationales du Français (CoVaNa-FR) as Resources for the Study of French from a Pluricentric Perspective. In Proceedings of the First Workshop on Applying NLP Tools to Similar Languages, Varieties and Dialects (Dublin, Ireland, August 23 2014). Stroudsburg: Association for Computational Linguistics: 48-57. En ligne à l'adresse suivante: http://www.aclweb.org/anthology/W/ W14/W14-5306.pdf. 